\title{
Aufbau von Arbeitgeberattraktivität - Identifikation der Determinanten und empirische Überprüfung
}
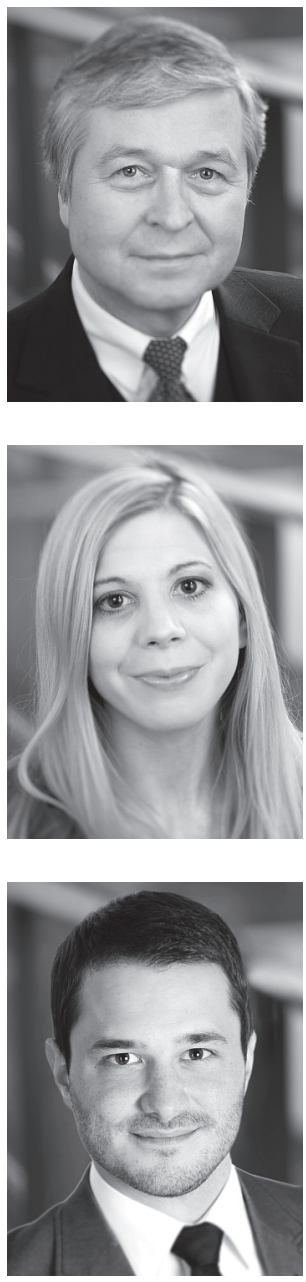

Manfred Brubn, Verena Batt, Benjamin Flückiger

Arbeitgeberattraktivität, Arbeitgeberimage, Arbeitsplatzqualität, Unternehmensimage, Skalenentwicklung, Strukturgleichungsanalyse

Employer Attractiveness, Employer Image, Quality in the Workplace, Corporate Image, Scale Development, Structural Equation Analysis

Die grosse Bedeutung qualifizierter Fach- und Führungskräfte für den Unternehmenserfolg sowie die sich abzeichnenden Veränderungen auf dem Arbeitsmarkt und in der demografischen Entwicklung machen es für Unternehmen verstärkt notwendig, auf dem Arbeitsmarkt als attraktiver Arbeitgeber wahrgenommen zu werden. Bislang wurden die Einflussfaktoren der Arbeitgeberattraktivität jedoch lediglich aus Sicht von potenziellen Mitarbeitenden untersucht; die aktuellen Mitarbeitenden im Unternehmen fanden bislang kaum Berücksichtigung. Das Ziel des Artikels besteht darin, die Attraktivitätsdeterminanten zu identifizieren und empirisch auf ihren Einfluss bei aktuellen Mitarbeitenden hin zu untersuchen. Die empirische Studie zeigt, dass die Arbeitgeberattraktivität bei Mitarbeitenden vor allem durch weiche Faktoren, wie z.B. den Führungsstil und die Arbeitsplatzqualität, sowie durch Imagefaktoren beeinflusst wird. Harte Faktoren, wie z.B. die Entlohnung oder Entwicklungsmöglichkeiten, üben im Rahmen der vorliegenden Untersuchung keinen signifikanten Einfluss auf die Attraktivitätswahrnehmung aus.

In view of the important contribution that technical specialists and managerial staff make in forging a company's success, and given emerging labor market and demographic developments, it is increasingly necessary for companies to be perceived as attractive employers on the job market. However, research, so far, has only studied factors that influence employer attractiveness from the prospective employee's point of view; actual employees have not been considerated. The objective of this article is to identify the major determinants of attractiveness and - from the perspective of actual employees - empirically investigate their effect. The empirical study shows that employer attractiveness is influenced mainly by soft factors, such as leadership style and workplace quality, as well as image factors. Hard factors, such as remuneration or career possibilities, do not have any significant influence on employer attractiveness. 


\section{Relevanz des Aufbaus von Arbeitgeberattraktivität}

Die Erkenntnis, dass Humankapital für die Wettbewerbsfähigkeit von Unternehmen von grosser Bedeutung ist, hat sich sowohl in der Forschung als auch in der Unternehmenspraxis durchgesetzt (Sutherland et al. 2002; Berthon et al. 2005; Roy 2008). So ist bekannt, dass es qualifizierte, motivierte und talentierte Mitarbeitende benötigt, um wichtige strategische Wettbewerbsvorteile erzielen zu können (Moroko/Uncles 2008; Krüger 2012). Aufgrund der zentralen Bedeutung der Mitarbeitenden für den Unternehmenserfolg herrscht auf dem Arbeitsmarkt ein reger Wettbewerb um fähige Nachwuchskräfte. In der wissenschaftlichen Literatur wurde dieser Wettbewerb, vor allem in Bezug auf so genannte High Potentials, bereits ausführlich unter dem Begriff „War for Talent“ bzw. „Battle for the talent" diskutiert (Chambers et al. 1998; Axelrod et al. 2001; Gaddam 2008).

Vor dem Hintergrund der aktuellen Wirtschafts- und Arbeitsmarktlage sprechen einige Faktoren dafür, dass die Wettbewerbsintensität für qualifiziertes und talentiertes Personal auch in Zukunft anhalten und sich durch den demografischen Wandel noch erheblich verschärfen wird (Heilmann 2010; Arachchige/Robertson 2011; Van Hoye/Saks 2011). Zudem ist ein Trend hin zu einer Wissensgesellschaft zu beobachten, wodurch die Anforderungen an die intellektuelle Qualifikation und Leistung der Mitarbeitenden stetig steigen (Ewing et al. 2002; Stotz/Wedel 2009). Durch die anhaltende Globalisierung, den Aufstieg von Emergent Economies, das Wachstum der bestehenden Märkte sowie neuer Industriesektoren und den daraus resultierenden komplexen und wissensintensiven Unternehmensaufgaben verstärkt sich einmal mehr die Nachfrage nach Erfolg bringenden Mitarbeitenden (Tochtermann/Abend 2003; Wiese 2005; Moroko/Uncles 2008).

Um die fachlich besten und zum Unternehmen passenden Mitarbeitenden zu finden und langfristig zu binden, gilt es, auf dem Arbeitsmarkt als attraktiver Arbeitgeber wahrgenommen zu werden (Sutherland et al. 2002; Moroko/Uncles 2009; Shah 2011; Forster et al. 2012). Dies ermöglicht es, von der Zielgruppe gegenüber den direkten Konkurrenten als bevorzugter Arbeitgeber, als Employer of Choice, wahrgenommen zu werden (Backhaus/Tikoo 2004; Stotz/Wedel 2009).

Angesichts der Bedeutung der Arbeitgeberattraktivität für die Mitarbeitergewinnung und -bindung ist die Kenntnis jener Faktoren notwendig, die zum Aufbau von Arbeitgeberattraktivität beitragen. Bei Betrachtung der existierenden wissenschaftlichen Literatur ist jedoch erkennbar, dass in den bisherigen Studien zur Untersuchung der Determinanten der Arbeitgeberattraktivität zwar eine Vielzahl von Einflussfaktoren aufgedeckt wird, deren Identifikation aber zumeist aus der Perspektive potenzieller Mitarbeitender, i.d.R. Studierender, erfolgt (z.B. Highhouse et al. 1999; Teufer 1999; Holtbrügge/Rygl 2002; Grobe 2003; Hinzdorf et al. 2003; Slaughter et al. 2004; Lievens et al. 2005; Burmann/Schäfer 2007; Kirchgeorg/Günther 2006; Roy 2008; Wilden et al. 2010; Arachchige/Robertson 2011; Böttger 2012). Ex-ante Untersuchungen zur Arbeitgeberattraktivität, wie sie Studierendenbefragungen darstellen, basieren jedoch nicht auf gesammelten Erfahrungen, sondern auf Vorstellungen, Vermutungen und Erwartungen betreffend bestimmter Unternehmen. Daher stimmen die aus der Sicht der potenziellen Mitarbeitenden identifizierten Attraktivitätstreiber nicht unbedingt mit denen aus der Sicht der aktuellen, bereits in Unternehmen tätigen Mitarbeitenden überein (Maxwell/Knox 2009). So weist z.B. die Studie von Lievens et al. (2007) nach, dass hinsichtlich der Einflussfaktoren der Arbeitsgeberattraktivität Unterschiede zwischen potenziellen und aktuellen Mitarbeitenden bestehen. 
Gerade bei den aktuellen Mitarbeitenden ist die Kenntnis der Determinanten der Attraktivitätswahrnehmung von Bedeutung, da sie als Botschafter des Unternehmens das Arbeitgeberimage nach aussen transportieren und dadurch Einfluss auf die Arbeitgeberwahrnehmung der potenziellen Mitarbeitenden nehmen. Eine der wenigen wissenschaftlichen Arbeiten, die sich mit den Attraktivitätstreibern aus Sicht bestehender Mitarbeitender beschäftigen, stellt die erwähnte Studie von Lievens et al. (2007) dar. In der Untersuchung wird sich mit der belgischen Armee jedoch auf lediglich einen einzigen Arbeitgeber konzentriert. Eine Generalisierbarkeit der Ergebnisse ist angesichts der Besonderheiten des Arbeitgebers in Frage zu stellen. Selbiges gilt für die Studien von Lievens/Highbouse (2003) und Lievens (2007), in denen Mitarbeitende einer Bank bzw. ebenfalls Armeeangehörige befragt werden. Praxisorientierte Arbeiten mit aktuellen Mitarbeitenden stellen die Studien des „Great place to work ${ }^{\circledR}$ Institutes“ (2012) sowie der Universum Communications AB (2012) dar. Die Studien im Kontext der „Great place to work“-Initiative beschäftigen sich allerdings weniger mit der Arbeitgeberattraktivität und deren Determinanten, sondern mit der erlebten Arbeitsplatzkultur. Im Rahmen der Universum Professional Surveys liegt der Fokus auf der Arbeitgeberattraktivität, weniger auf deren Einflussgrössen. Insofern ist auch in diesen Studien nur ein begrenzter Hinweis auf die Determinanten der Arbeitgeberattraktivität bei aktuellen Mitarbeitenden in Unternehmen zu finden.

Zusammenfassend machen die Ausführungen deutlich, dass bislang weitgehende Unwissenheit über jene Variablen einhergeht, die aus Sicht von aktuellen Mitarbeitenden im Unternehmen Einfluss auf die Arbeitgeberattraktivität nehmen. Vor diesem Hintergrund besteht die Zielsetzung des vorliegenden Artikels darin, die aktuelle Diskussion zur Arbeitgeberattraktivität um die noch unzureichend erforschte Perspektive aktueller Mitarbeitender in Unternehmen zu erweitern. Der Fokus liegt dabei auf der Identifikation derjenigen Determinanten, die aus Sicht der Mitarbeitenden für die Entstehung von Arbeitgeberattraktivität verantwortlich sind. Die Kenntnis über die relevanten Einflussfaktoren der Arbeitgeberattraktivität liefert Unternehmen wertvolle Informationen für eine Vielzahl personalpolitischer Entscheidungen.

Die Struktur des Artikels gliedert sich in vier Kapitel. Das zweite Kapitel widmet sich dem Aufbau des Untersuchungsmodells. Hierfür wird zunächst auf den Begriff der Arbeitgeberattraktivität sowie auf die darauf basierende Konzeptualisierung eingegangen. Auf Basis einer umfassenden Literatursichtung werden anschliessend mögliche Einflussgrössen der Arbeitgeberattraktivität abgeleitet. Dies mündet in die Entwicklung des Untersuchungsmodells und die Hypothesenformulierung. Im dritten Kapitel erfolgt die Operationalisierung der Konstrukte. Basierend auf einer Befragung mit aktuellen Mitarbeitenden in Unternehmen werden anschliessend die postulierten Determinanten der Arbeitgeberattraktivität anhand eines Strukturgleichungsmodells überprüft. Das vierte Kapitel bildet schliesslich das Fazit mit Implikationen für die Unternehmenspraxis sowie Hinweisen für die weiterführende Forschung.

\section{Untersuchungsmodell zum Aufbau der Arbeitgeberattraktivität}

\subsection{Begriff und Konzeptualisierung der Arbeitgeberattraktivität}

Das Konzept der Arbeitgeberattraktivität wurde in der Literatur bislang vor allem im Zusammenhang mit der Berufswahl von potenziellen Arbeitnehmern betrachtet (z.B. Soutar/ Clarke 1983; Lievens/Highhouse 2003; Berthon et al. 2005; Lievens et al. 2005; Roy 
2008). So verstehen Berthon et al. $(2005,156)$ unter der Arbeitgeberattraktivität „the envisioned benefits that a potential employee sees in working for a specific organisation ". Wie aus dieser Begriffsbestimmung hervorgeht, spiegelt die Arbeitgeberattraktivität die positiven Assoziationen bzw. Nutzenmerkmale, die ein potenzieller Arbeitnehmer mit dem Unternehmen als Arbeitgeber verbindet, wider. Dieses arbeitgeberbezogene Vorstellungsbild wird als Arbeitgeberimage bezeichnet (Petkovic 2008). Die Arbeitgeberattraktivität ergibt sich somit direkt aus dem Arbeitgeberimage. Sie stellt die positive Bewertung des Arbeitgeberimages durch die Zielgruppe dar. Je positiver die Assoziationen bezüglich des Images eines Arbeitgebers sind, desto attraktiver ist dieser in den Augen der Zielgruppe.

Die Arbeitgeberattraktivität wird im vorliegenden Artikel nicht aus Sicht potenzieller, sondern aus der Perspektive bereits agierender (aktueller) Arbeitnehmer betrachtet. Die positiven Assoziationen und Nutzenmerkmale, die von Seiten des Arbeitnehmers bei Vorliegen von Arbeitgeberattraktivität mit dem Unternehmen verbunden werden, sind als so genannte Attraktivitätsmerkmale anzusehen. Nehmen Mitarbeitende ihren Arbeitgeber als attraktiv wahr, so arbeiten sie gerne bei diesem, weisen ihm gegenüber eine positive Einstellung auf und ziehen ihn gegenüber anderen, vergleichbaren Arbeitgebern vor. Als zentrale Attraktivitätsmerkmale lassen sich demnach gerne arbeiten, Präferenz und eine positive Einstellung gegenüber dem Arbeitgeber subsumieren. Die emotionale Verbundenheit mit dem Arbeitgeber ist als weiteres Attraktivitätsmerkmal anzusehen (zur Ableitung der Attraktivitätsmerkmale Lievens et al. 2005; Barrow/Mosley 2006; Davies 2008).

\subsection{Identifikation von Einflussfaktoren der Arbeitgeberattraktivität und Hypothesengenerierung}

Zur Identifikation der relevanten Einflussfaktoren der Attraktivität von Arbeitgebern wurden konzeptionelle und empirische Studien gesichtet. Aufgrund mangelnder Untersuchungen mit aktuellen Mitarbeitenden war es notwendig, auf Studien zurückzugreifen, die den Fokus auf potenzielle Arbeitnehmer legten (z.B. Turban/Keon 1993; Simon et al. 1995; Highbouse et al. 1999; Teufer 1999; Cable/Graham 2000; Lievens et al. 2001; Lievens/ Highhouse 2003; Berthon et al. 2005; Schulze et al. 2005; Lievens et al. 2005; Kirchgeorg/Günther 2006; Knox/Freeman 2006; Schwaab 2008; Trost 2008; Zirnsack 2008; Moroko/Uncles 2009; Arachchige/Robertson 2011; Böttger 2012). Die Ausnahme stellen die bereits zitierten Studien von Lievens/Highhouse (2003), Lievens (2007) und Lievens et al. (2007) dar. Jene Attraktivitätsdeterminanten, denen in den Studien eine hohe Bedeutung zugewiesen wurde, erfuhren eine weitere Berücksichtigung. Im Folgenden wird auf die identifizierten Einflussfaktoren der Arbeitgeberattraktivität eingegangen, und es werden Hypothesen generiert.

Das direkte Arbeitsumfeld bzw. die Arbeitsbedingungen werden in der Literatur als wesentliche Einflussfaktoren der Arbeitgeberattraktivität angesehen. In diesem Zusammenhang ist die Arbeitsplatzqualität von Bedeutung. Merkmale einer hohen Arbeitsplatzqualität sind z.B. attraktive Arbeitszeitmodelle, wie die Möglichkeit zur flexiblen Gestaltung des Arbeitspensums. Der Spassfaktor bei der Arbeit ist in diesem Zusammenhang ebenfalls zu nennen. Zudem sind eine ausgewogene Work-Life-Balance und eine hohe Arbeitsplatzsicherheit bedeutungsvolle Aspekte der Arbeitsplatzqualität (z.B. Lievens/Highhouse 2003; Roy 2008). Dies führt zu folgender Hypothese:

$H_{1}$ : Die wahrgenommene Arbeitsplatzqualität beeinflusst die Arbeitgeberattraktivität direkt positiv. 
Aspekte des Führungsstils haben ebenfalls einen Einfluss auf die Arbeitgeberattraktivität. Neben dem Führungsstil selbst sind die interne Informations- und Kommunikationspolitik, die Leistungswertschätzung durch die Vorgesetzten und die Funktion der Vorgesetzten als Vorbild für Mitarbeitende als zentrale Aspekte anzufügen (z.B. Berthon et al. 2005; Knox/Freeman 2006; Petkovic 2008). Folgende Hypothese lässt sich formulieren:

$\mathrm{H}_{2}$ : Der wahrgenommene Führungsstil beeinflusst die Arbeitgeberattraktivität direkt positiv.

Neben den Faktoren, die die Arbeitsplatzqualität und den Führungsstil betreffen, hat die Entlohnung einen Einfluss auf die Arbeitgeberattraktivität. Neben dem (Einstiegs-)Gehalt sind hier das Lohnerhöhungspotenzial, Fringe Benefits (Lohnnebenleistungen im Sinne von überobligatorischen Sozialleistungen und Bruttolöhnen/-gehältern in Form von Sachleistungen und eine eventuelle Beteiligung am Erfolg des Unternehmens), beispielsweise in Form eines Bonussystems, von Bedeutung (Gillies/Jung 1999; Grobe 2003; Petkovic 2008). Dies führt zu folgender Hypothese:

$\mathrm{H}_{3}$ : Die wahrgenommene Entlohnung beeinflusst die Arbeitgeberattraktivität direkt positiv.

Als Attraktivitätsdeterminante ist ebenfalls das Stellenprofil anzusehen. So ist bezüglich der Gestaltung der Aufgabe bzw. der Tätigkeit im Unternehmen von grosser Bedeutung, wie interessant, vielfältig und herausfordernd diese betrachtet wird. Auch spielen die den Mitarbeitenden zugestandenen Entscheidungsbefugnisse eine Rolle (Berthon et al. 2005; Knox/Freeman 2006; Petkovic 2008; Maxwell/Knox 2009). Dies ergibt folgende Hypothese:

\section{$H_{4}$ : Das wahrgenommene Stellenprofil beeinflusst die Arbeitgeberattraktivität direkt po- sitiv.}

Die Entwicklungsmöglichkeiten, die die zukunftsgerichtete, unternehmensseitige Förderung der Mitarbeitenden widerspiegeln, zählen ebenfalls zu bedeutenden Einflussfaktoren der Arbeitgeberattraktivität. Hierunter lassen sich Karriere- und Aufstiegsaussichten, Ausund Weiterbildungsmöglichkeiten sowie die Lernchancen des Mitarbeitenden subsumieren. Auch wird der Möglichkeit, verschiedene Bereiche des Unternehmens kennenzulernen und in verschiedenen Bereichen zu arbeiten (interne Jobrotation), einen Einfluss auf die Arbeitgeberattraktivität zugesprochen (Berthon et al. 2005; Knox/Freeman 2006; Lievens 2007; Arachchige/Robertson 2011). Folgende Hypothese wird aufgestellt:

\section{$H_{5}$ : Die wahrgenommenen Entwicklungsmöglichkeiten beeinflussen die Arbeitgeberat- traktivität direkt positiv.}

Neben den bisher herausgearbeiteten Attraktivitätsdeterminanten werden in der Literatur auch solche Einflussgrössen als relevant betrachtet, die auf Imageaspekte des Unternehmens Bezug nehmen (z.B. Grosse Halbuer 2003; Roy 2008). Das entsprechende Konstrukt wird im vorliegenden Artikel als Unternehmensimage bezeichnet. Das Image spiegelt die Assoziationen wider, die von Mitarbeitenden mit dem Unternehmen verbunden werden (vgl. hierzu z.B. Keller 1993). Darunter zählen z.B. das Image der Produkte und Dienstleitungen, die das Unternehmen produziert, oder das Image der Branche, in der das Unternehmen tätig ist. Das Image reflektiert ausserdem, wie angesehen oder prestigeträchtig ein 
Unternehmen wahrgenommen wird. Das Ansehen eines Unternehmens in der Öffentlichkeit hat durch die Referenzwirkung und das verbundene Sozialprestige eine grosse Wirkung auf die Arbeitgeberwahrnehmung. Die Beurteilung des Unternehmensstandorts trägt ebenfalls zum Bild, das Mitarbeitende von einem Unternehmen haben, bei. Neben generellen Aspekten der Mobilität und des Arbeitsweges ist für die Imagebildung auch von Bedeutung, welche Möglichkeiten das Unternehmen zur internationalen Tätigkeit bietet. Ferner sind die Corporate Social Responsibility, die Kultur und die Werte, die das Unternehmen vertritt, sowie der Bekanntheitsgrad und die Glaubwürdigkeit des Unternehmens von Relevanz (Petkovic 2008). Dies führt zur Formulierung folgender Hypothese:

\section{$H_{6}$ : Das wahrgenommene Unternehmensimage beeinflusst die Arbeitgeberattraktivität di-} rekt positiv.

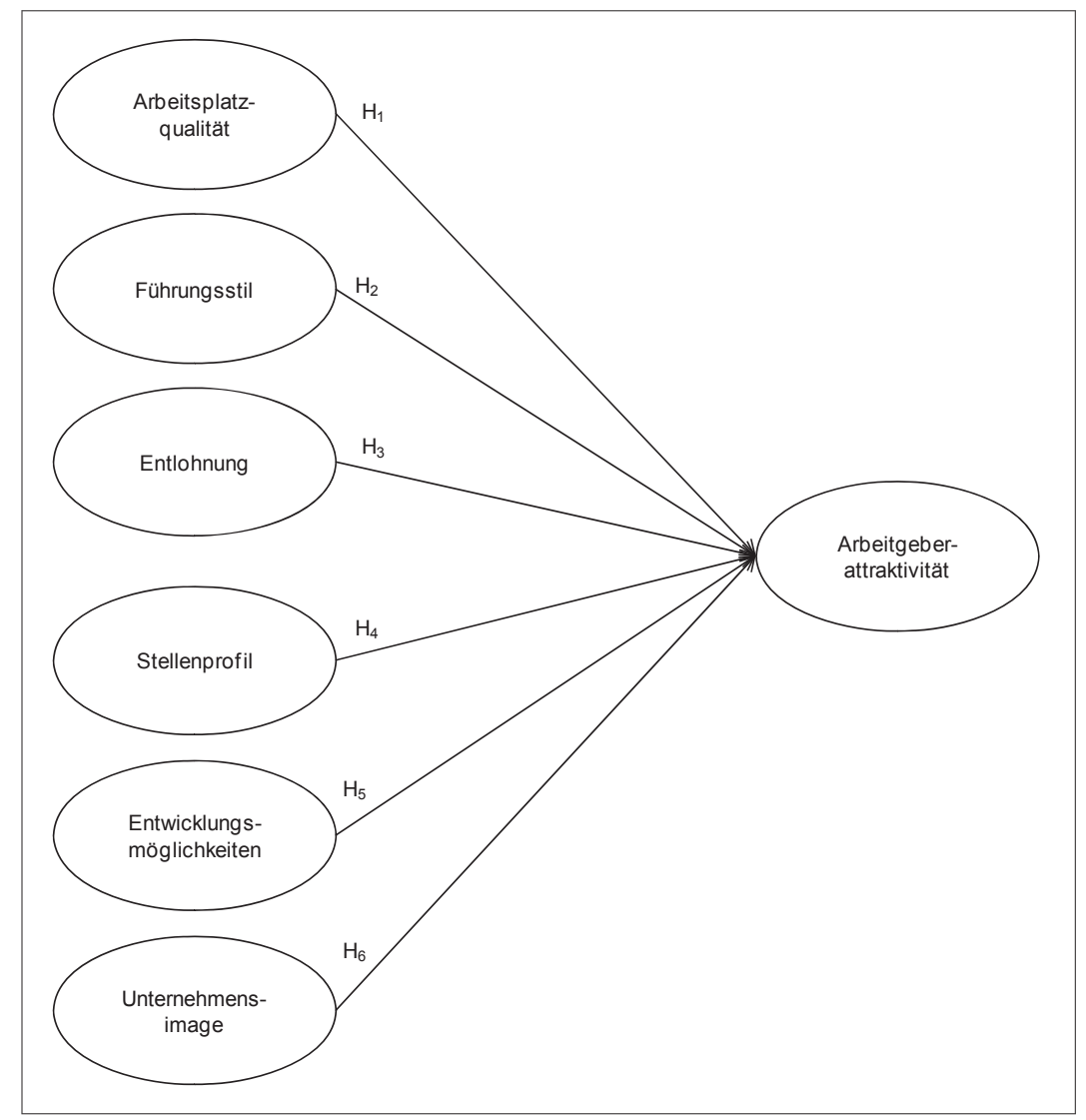

Abbildung 1: Untersuchungsmodell zum Aufbau der Arbeitgeberattraktivität

Zusammenfassend ergibt sich aus der Literaturdurchsicht die Identifikation von sechs Faktoren, die als die Arbeitgeberattraktivität bestimmend angesehen werden. Abbildung 1 zeigt die Wirkungszusammenhänge im Untersuchungsmodell auf. 


\section{Operationalisierung der Konstrukte und empirische Ergebnisse}

\subsection{Design und Durchführung der empirischen Untersuchung}

Die Frage, ob die identifizierten Grössen bei aktuellen Mitarbeitenden tatsächlich zur Entstehung der Arbeitgeberattraktivität beitragen, wird auf Basis einer empirischen Untersuchung beantwortet. Als Datenerhebungsmethode diente eine Befragung anhand eines standardisierten Fragebogens. Aufgrund der Effizienz der Datenerhebung und der einfachen Erreichbarkeit einer breiten Masse wurde eine computergestützte Online-Befragung gewählt. Die Umfrage wurde zum einen auf eine Online-Plattform gestellt. Dadurch wurde ein einfacher Zugriff auf den Fragebogen via Webbrowser gewährleistet. Um die Reichweite der Befragung zu fördern, wurde der Fragebogen zum anderen per Link zur Umfrage via Email und gemäss des „Schneeball-Prinzips“ (Zinkhan et al. 1983) an Teilnehmer verschiedener sozialer Netzwerke versendet. Im Gegensatz zu früheren Studien wurde dabei die Ansprache von Studierenden vermieden.

Der Zeitraum der Datenerhebung erstreckte sich auf das Jahr 2009. Insgesamt wurden bis zum Ende des Erhebungszeitraums 117 vollständige Datensätze generiert. 59,8 Prozent davon hatten bis zu fünf Jahren, 16,2 Prozent sechs bis zehn Jahre, 4,3 Prozent elf bis zwanzig Jahre und 19,7 Prozent mehr als zwanzig Jahre Berufserfahrung.

\subsection{Operationalisierung der Modellvariablen}

Für die Arbeitgeberattraktivität und deren Einflussfaktoren liegen keine geeigneten Messinstrumente vor, so dass für deren Messung die Entwicklung eigener Skalen notwendig war. Hierfür erfolgte eine Orientierung an der C-OAR-SE-Prozedur von Rossiter (2002). Das Verfahren wird gegenüber dem kennziffernorientierten Churchill-Prozess (Churchill 1979) als ein flexiblerer und offenerer Ansatz zur Entwicklung von Messskalen betrachtet (vgl. hierzu ausführlich Diamantopoulos 2005; Finn/Kayande 2005). Mit der Anwendung von C-OAR-SE wird die Identifikation der inhaltlichen Bestandteile der Modellkonstrukte gewährleistet (vgl. hierzu auch Rossiter 2002).

Rossiter (2002, 305ff.) schlägt für die Entwicklung von Messinstrumenten sechs Schritte vor. Im ersten Schritt ist das zu untersuchende Konstrukt in Bezug auf das Objekt, das Attribut und die Beurteiler zu definieren. Tabelle 1 gibt einen Überblick über die Definitionen der hier interessierenden Modellkonstrukte.

Im zweiten Schritt erfolgt die Klassifizierung des Objekts der jeweiligen Konstrukte (Rossiter 2002). Objekte können vom Typ „,concrete singular“ (fast alle Beurteiler kennen das Objekt, beschreiben es ähnlich und sehen es als einzeln an), „abstract collective“ (Objekt ist aus Sicht der Beurteiler heterogen und hat verschiedene Bestandteile) oder „abstract formed“ (Objekt ist aus Sicht der Beurteiler heterogen und hat verschiedene Dimensionen) sein. Für die Klassifizierung des Objekts wurden zehn Interviews mit Arbeitnehmern unterschiedlicher Arbeitgeber aus verschiedenen Branchen durchgeführt. Die Probanden wurden gefragt, was sie sich unter dem Begriff Arbeitgeber vorstellen. Es zeigte sich, dass damit stets der jeweilige Arbeitgeber des Probanden verbunden wird. Da es sich dabei jeweils um ein einzelnes Unternehmen in der Funktion als Arbeitgeber handelt, sind die Objekte im vorliegenden Fall vom Typ „concrete singular“.

Im dritten Schritt erfolgt die Klassifizierung des Attributs (Rossiter 2002). Attribute können unterschiedlicher Art sein. Sind sie vom Typ „concrete“, so bedeutet dies, dass nahezu alle Beurteiler das Attribut als ähnlich beschreiben, mit der Folge, dass die Messung 


\begin{tabular}{|l|l|}
\hline Konstruktbezeichnungen & Definition der Konstrukte nach C-OAR-SE \\
\hline Arbeitsplatzqualität & $\begin{array}{l}\text { Die wahrgenommene Arbeitsplatzqualität [Attribut] eines Ar- } \\
\text { beitgebers [Objekt] aus Sicht von dessen Mitarbeitenden [Be- } \\
\text { urteiler] }\end{array}$ \\
\hline Führungsstil & $\begin{array}{l}\text { Der wahrgenommene Führungsstil [Attribut] eines Arbeitge- } \\
\text { bers [Objekt] aus Sicht von dessen Mitarbeitenden [Beurteiler] }\end{array}$ \\
\hline Entlohnung & $\begin{array}{l}\text { Die wahrgenommene Entlohnung [Attribut] eines Arbeitgebers } \\
\text { [Objekt] aus Sicht von dessen Mitarbeitenden [Beurteiler] }\end{array}$ \\
\hline Stellenprofil & $\begin{array}{l}\text { Das wahrgenommene Stellenprofil [Attribut] eines Arbeitge- } \\
\text { bers [Objekt] aus Sicht von dessen Mitarbeitenden [Beurteiler] }\end{array}$ \\
\hline Entwicklungsmöglichkeiten & $\begin{array}{l}\text { Die wahrgenommenen Entwicklungsmöglichkeiten [Attribut] } \\
\text { eines Arbeitgebers [Objekt] aus Sicht von dessen Mitarbeiten- } \\
\text { den [Beurteiler] }\end{array}$ \\
\hline Unternehmensimage & $\begin{array}{l}\text { Das wahrgenommene Unternehmensimage [Attribut] eines Ar- } \\
\text { beitgebers [Objekt] aus Sicht von dessen Mitarbeitenden [Be- } \\
\text { urteiler] }\end{array}$ \\
\hline Arbeitgeberattraktivität & $\begin{array}{l}\text { Die wahrgenommene Attraktivität [Attribut] eines Arbeitge- } \\
\text { bers [Objekt] aus Sicht von dessen Mitarbeitenden [Beurteiler] }\end{array}$ \\
\hline
\end{tabular}

Tabelle 1: Konstruktdefinitionen im Überblick

über ein einzelnes Item ausreicht (Single-Item-Messung). Liegt ein Attribut vom Typ „formed" vor, so formen verschiedene Komponenten das Attribut. Folglich ist eine formative Konstruktmessung notwendig. Bei einem Attribut vom Typ „eliciting“ bedingt das Attribut die Items, d.h. die Items sind nicht definierend, sondern stellen Manifestationen des Konstrukts dar. Dies führt zu einer reflektiven Konstruktoperationalisierung. Zur Klassifizierung der Attribute dienten die bereits erwähnten Interviews. Die Probanden wurden gefragt, welche Merkmale sie mit den einzelnen Attributen, z.B. der wahrgenommenen Entlohnung, verbinden. Es wurde deutlich, dass in der Wahrnehmung der Probanden jedes Attribut durch verschiedene Indikatoren manifestiert wird. Die wahrgenommene Entlohnung im Unternehmen wird z.B. aus Sicht der Befragten durch die Indikatoren wahrgenommener (Einstiegs-) Lohn, wahrgenommenes Lohnerhöhungspotenzial sowie wahrgenommene Erfolgsbeteiligung und Fringe Benefits widergespiegelt. Bei sämtlichen Konstrukten liegen somit Attribute vom Typ „eliciting“ vor. Dies indiziert eine reflektive Konstruktmessung (vgl. zur formativen vs. reflektiven Konstruktoperationalisierung ausführlich Chin 1998a; Diamantopoulos/Winklhofer 2001; Jarvis et al. 2003; Chin et al. 2008).

Die Beurteilung von Objekt und Attribut hängt jeweils von den Betrachtern bzw. Beurteilern ab. In einem vierten Schritt ist daher eine genaue Definition des Beurteilers notwendig. Beurteiler können „Individuen“ (bei self-reports), „Experten“ oder „Group raters“ (sample von z.B. Konsumenten oder Mitarbeitenden) sein (Rossiter 2002). Im vorliegenden Fall sind die Beurteiler vom Typ „group raters“, da es sich bei ihnen um ein Sample von Mitarbeitenden handelt, die ihren Arbeitgeber anhand der jeweiligen Attribute beurteilen. 
Im fünften Schritt erfolgt - durch die Kombination der Objekt- und Attributsbestandteile - die Skalenbildung. Einen Überblick über die zu den hier betrachteten Konstrukten gehörigen Indikatoren gibt Tabelle 2 im Anhang.

Im sechsten Schritt des C-OAR-SE-Verfahrens erfolgt die Berechnung eines Gesamtwerts für ein Konstrukt (Rossiter 2002). Je nach Art der Objekte und/oder der Attribute werden Indizes, Durchschnitts- oder Einzelwerte berechnet. Da es sich im vorliegenden Fall um Objekte vom Typ „concrete singular“ und Attribute vom Typ „eliciting“ handelt, wurde im Rahmen der Datenanalyse (vgl. Abschnitt 3.3) für jedes Konstrukt eine Mittelwertberechnung über alle Konstruktitems vorgenommen.

In weiteren fünf Experteninterviews wurden die entwickelten Skalen inhaltlich auf Vollständigkeit, Redundanz und Verständlichkeit überprüft. Es handelte sich bei den Befragten um Beschäftigte, die im Arbeitgebermarketing verschiedener Unternehmen tätig sind und dadurch ein umfassendes Fachwissen auf dem Gebiet der Arbeitgeberattraktivität aufweisen. Die Operationalisierung der Konstrukte wurde von den Experten als sinnvoll bestätigt. Es wurden keine fehlenden Inhalte aufgedeckt und alle Indikatoren als verständlich bestätigt.

Im Anschluss an die Konstruktoperationalisierung wurde der Fragebogen entwickelt. Dieser enthielt Fragestellungen, die sich nach den einzelnen Indikatoren der zu messenden Konstrukte richteten. Um zu überprüfen, wie der Proband die Attraktivität seines Arbeitgebers bewertet, wurden Aussagen formuliert, die danach zu beurteilen sind, wie gut sie auf ihn bzw. seinen Arbeitgeber zutreffen. Zur Beurteilung der Aussagen diente eine zweipolige, siebenstufige Ratingskala. Als Extrema wurden die Formulierungen „1 - Trifft überhaupt nicht zu“ bis „7 - Trifft voll und ganz zu“ gewählt.

\subsection{Schätzung des Kausalmodells und Modellbeurteilung}

Für die Überprüfung des konzeptualisierten Untersuchungsmodells wurde eine Strukturgleichungsanalyse mithilfe des Analyseprogramms PLS Graph 3.0 durchgeführt. Der Rückgriff auf den varianzbasierten PLS-Ansatz erfolgte zum einen aufgrund der geringen Stichprobengrösse. Zum anderen erweisen sich varianzbasierte Verfahren gegenüber kovarianzbasierten Ansätzen insbesondere dann als vorteilhaft, wenn das Augenmerk, wie im vorliegenden Fall, auf der bestmöglichen Vorhersage des Zielkonstrukts und weniger auf das Testen eines theoretisch hergeleiteten Hypothesengefüges gerichtet ist (Herrmann et al. 2006). Zur Abschätzung der Modellgüte, und damit zur Sicherstellung der Zuverlässigkeit des Gesamtmodells, wurde sich an den von Chin vorgeschlagenen Prüfkriterien orientiert (Chin 1998b; Chin/Newsted 1999; vgl. für eine Übersicht über die Prüfkriterien für PLSModelle, Hermann et al. 2006).

Im Hinblick auf die Messmodelle kommt zunächst eine exploratorische Faktorenanalyse zum Einsatz. Es zeigt sich, dass - gemäss der Konzeptualisierung - bei sämtlichen Konstrukten exakt ein Faktor extrahiert wird, der zudem mehr als die geforderten 50 Prozent der Varianz der ihm zugehörigen Indikatoren erklärt (Homburg/Giering 1996). Das Cronbach'sche Alpha der Konstrukte liegt über dem Schwellenwert von 0,7 und verweist damit auf hohe interne Konsistenz (Nunnally 1978). Eine Ausnahme ist das Konstrukt Arbeitsplatzqualität. Die Unterschreitung ist jedoch mit einem Wert von 0,669 nur geringfügig und wird daher toleriert. Die Konstruktreliabilität überschreitet jeweils den geforderten Mindestwert von 0,6 (Bagozzi/Yi 1988). Die durchschnittlich erfasste Varianz liegt stets über dem Schwellenwert von 0,5 (Fornell/Larcker 1981). Im Hinblick auf die Faktorla- 
dungen der Konstrukte liegen einige Werte unter dem geforderten Mindestwert von 0,7, dies aber meist geringfügig, so dass nahezu sämtliche Items beibehalten werden. Die Ausnahme stellen die Indikatoren Internationalität und Bekanntheitsgrad des Konstrukts Unternehmensimage dar. Aufgrund der sehr niedrigen Werte der Faktorladungen werden diese beiden Items eliminiert. Der erforderliche T-Wert von 1,64 kann für alle Items beobachtet werden. Die Überprüfung des Fornell-Larcker-Kriteriums zeigt schliesslich das Vorliegen von Diskriminanzvalidität.

Im Rahmen der Beurteilung des Strukturmodells erfolgt schliesslich die Überprüfung der postulierten Hypothesen. Die Pfadkoeffizienten und die entsprechenden T-Werte geben hierbei Auskunft über die Einflussstärke der vermuteten Determinanten auf die Arbeitgeberattraktivität. Wie aus Abbildung 2 hervorgeht, sind - abgesehen vom nicht zu bestätigenden Einfluss der Entwicklungsmöglichkeiten bzw. der Entlohnung auf die Arbeitgeberattraktivität - sämtliche Pfadzusammenhänge signifikant. Dabei erweisen sich die positive Wahrnehmung des Führungsstils $(0,364)$ und das positiv bewertete Unternehmensimage $(0,256)$ am bedeutendsten für die Entstehung der Arbeitgeberattraktivität. Die Wahrnehmung einer hohen Arbeitsplatzqualität hat mit einem Pfadkoeffizienten von 0,203 den drittgrössten Einfluss auf die Attraktivitätswahrnehmung des Arbeitgebers, gefolgt von der positiven Wahrnehmung des Stellenprofils $(0,131)$. Zudem verweist das $\mathrm{R}^{2}$ von $0,688 \mathrm{da}-$ rauf, dass die Arbeitgeberattraktivität „substanziell“ (Chin 1998b, 323) von den exogenen

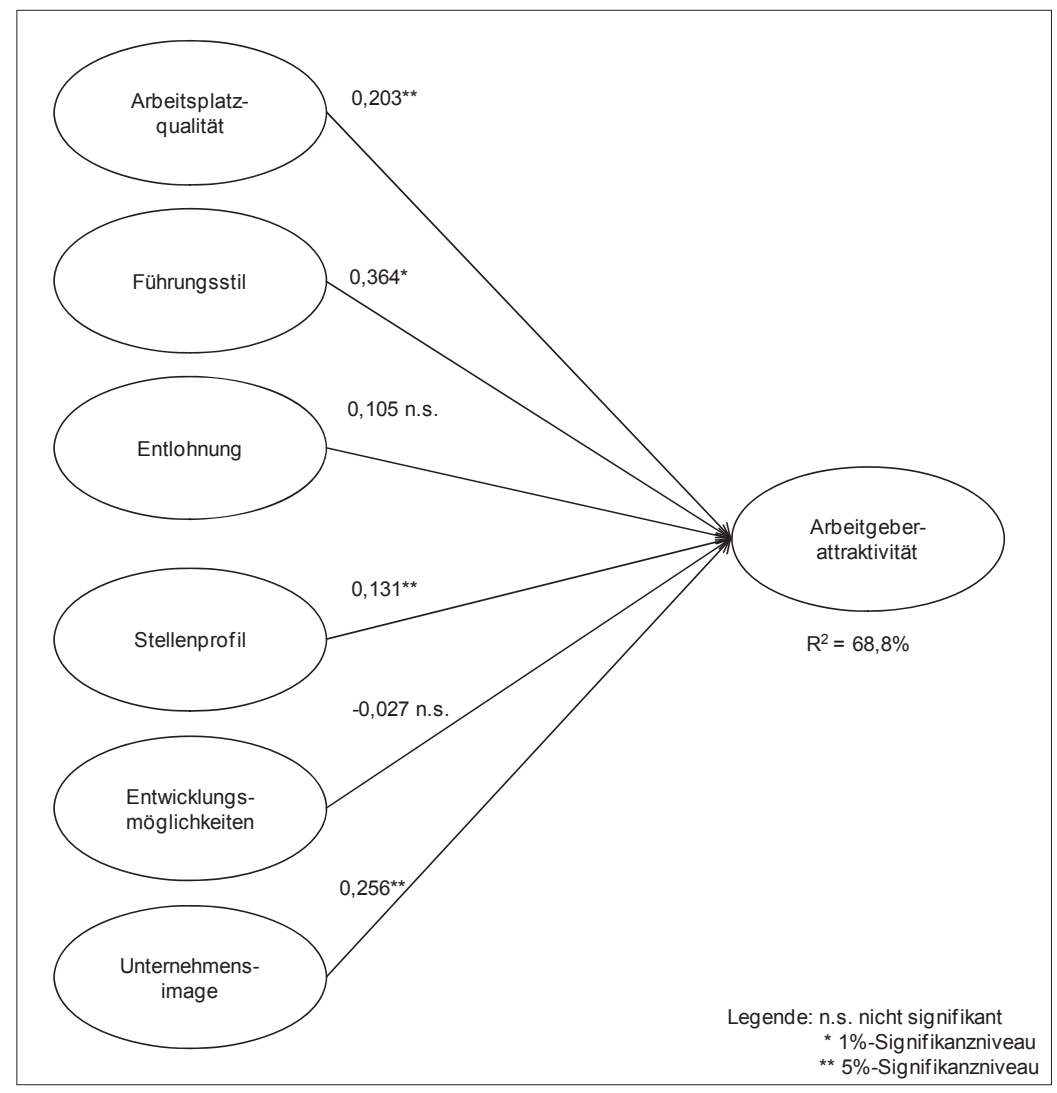

Abbildung 2: Ergebnisse des Strukturgleichungsmodells 
Konstrukten erklärt wird. Das zeigt, dass gut zwei Drittel der Grössen, die für den Aufbau einer Arbeitgeberattraktivität verantwortlich sind, identifiziert wurden. Abbildung 2 liefert einen Überblick über die Ergebnisse der Modellprüfung.

Da im Rahmen der Studie sämtliche Variablen von jeweils einer Person beurteilt wurden, besteht die Gefahr eines Common-Method-Bias (CMB) (Podsakoff et al. 2003). Die Durchführung des Harman's One-Factor-Tests zeigt jedoch, dass kein Faktor durch einen Grossteil der Kovarianz erklärt wird (Harman 1967). Darüber hinaus wurde ein Common Method Factor mit sämtlichen Konstruktindikatoren in das Gesamtmodell eingeführt und in Beziehung zu den Modellkonstrukten gesetzt. Die Analyse zeigt, dass die Faktorladungen des Common Method Factors meist nicht signifikant sind. Die quadrierten Ladungen des Faktors sind deutlich geringer als die der übrigen Konstrukte. Dies verweist darauf, dass in der vorliegenden Stichprobe kein CMB besteht (Liang et al. 2007).

\subsection{Hypothesenprüfung}

Im Rahmen der kausalanalytischen Prüfung des Modells konnten vier der sechs aufgestellten Hypothesen bestätigt werden. Vor dem Hintergrund der Analyse lassen sich folgende Ergebnisse festhalten:

Die empirischen Ergebnisse zeigen, dass eine hohe Qualitätswahrnehmung des Arbeitsplatzes (z.B. Work-Life-Balance, positives Arbeitsklima) die Attraktivität des Arbeitgebers in der Wahrnehmung des Mitarbeitenden steigen lässt. Somit ist Hypothese $H_{1}$ zu bestätigen.

Hypothese $\mathrm{H}_{2}$ findet ebenfalls empirischen Nachweis. Je positiver der Führungsstil beurteilt wird, desto attraktiver wird der Arbeitgeber eingeschätzt. Der Führungsstil stellt die wichtigste Determinante der Arbeitgeberattraktivität dar. Für die Schaffung von Arbeitgeberattraktivität ist somit dem Verhalten der Führungskräfte besondere Beachtung zu schenken.

Hypothese $H_{3}$ kann nicht bestätigt werden. Arbeitgeber, die in Verbindung mit einem attraktiven Entlohnungssystem gebracht werden, werden dadurch nicht zwingend als attraktiv wahrgenommen. Statistische Erkenntnisse (z.B. zu geringe Standardabweichungen) liefern keine Erklärung für das überraschende Ergebnis. Möglicherweise ist der Zusammenhang zwischen den Konstrukten jedoch von Faktoren, wie z.B. dem Alter der Mitarbeitenden, abhängig. In einer Studie konnte z.B. nachgewiesen werden, dass materielle Aspekte, wie beruflicher Status, Wettbewerb und Aufstiegsorientierung, für ältere Arbeitnehmer weniger von Bedeutung sind als für jüngere Mitarbeitende (Brinkmann 2007). Dies ist dementsprechend auch für die Entlohnung anzunehmen. Daher ist der nicht nachgewiesene Wirkungszusammenhang möglicherweise auf einen ausgeprägten Anteil an älteren Arbeitnehmern im Datensatz zurückzuführen. Darauf verweist auch die deskriptive Analyse der Stichprobe. So hat knapp ein Fünftel der Befragten mehr als 20 Jahre Berufserfahrung und dementsprechend vermutlich bereits ein höheres Lebensalter erreicht (vgl. Abschnitt 3.1).

Empirischen Nachweis erfährt Hypothese $H_{4}$. Es wird nachgewiesen, dass ein attraktives Stellenprofil die Arbeitgeberattraktivität positiv beeinflusst. Auf diese Determinante ist bei der Ausgestaltung von Massnahmen zur Steigerung von Arbeitgeberattraktivität somit ebenfalls das Augenmerk zu legen.

Hypothese $\mathrm{H}_{5}$ ist hingegen abzulehnen. Die wahrgenommenen Entwicklungsmöglichkeiten nehmen keinen Einfluss auf die Attraktivität des Arbeitgebers. Wie bei Hypothese $\mathrm{H}_{3}$ 
ist eine Ursache für den fehlenden empirischen Nachweis in der Existenz von Faktoren, die Einfluss auf die Stärke des Wirkungszusammenhangs nehmen, zu sehen. In diesem Zusammenhang ist auf die oben genannte Studie (Brinkmann 2007) hinzuweisen. So wird für die Stichprobe ein hoher Anteil älterer Mitarbeitender, für die die Entwicklungsmöglichkeiten im Unternehmen von geringerer Bedeutung sind als für jüngere Arbeitnehmer, vermutet.

Werden Imagemerkmale (z.B. Glaubwürdigkeit, Unternehmenskultur, Standort) des arbeitgebenden Unternehmens von den Mitarbeitenden als positiv bewertet, so hat dies einen direkten positiven signifikanten Einfluss auf die Attraktivitätswahrnehmung des Arbeitgebers. Damit bestätigt sich Hypothese $H_{6}$. Zurückzuführen ist die Bedeutung dieser Einflussgrösse auf die Möglichkeit von Arbeitnehmern, mit den positiven Merkmalen eines Unternehmens, wie z.B. Unternehmenserfolg oder Glaubwürdigkeit, nach aussen hin „Impression Management" zu betreiben und dadurch die Wahrnehmung seines Selbst zu erhöhen. Imagefaktoren sind somit für die Attraktivitätswahrnehmung von hoher Bedeutung.

Insgesamt zeigt sich, dass mit einer Varianzerklärung von knapp 70 Prozent ein grosser Teil der Attraktivitätsdeterminanten identifiziert wurde. Es offenbart sich, dass es bei Mitarbeitenden neben Imagefaktoren vor allem „weiche“ Faktoren sind, wie z.B. die Arbeitsplatzqualität oder der Führungsstil, die für den Aufbau von Arbeitgeberattraktivität verantwortlich sind. „Harte“ Faktoren, wie z.B. die Entlohnung oder Entwicklungsmöglichkeiten im Unternehmen, beeinflussen die Attraktivitätswahrnehmung den Untersuchungsergebnissen zufolge nicht.

\section{Fazit, Implikationen und Ausblick}

Ausgangspunkt dieses Artikels war die Erkenntnis, dass für Unternehmen aufgrund der zunehmenden Bedeutung qualifizierter Mitarbeitender verstärkt die Notwendigkeit besteht, auf dem Arbeitsmarkt als attraktiver Arbeitgeber wahrgenommen zu werden. Die Relevanz der Arbeitgeberattraktivität wird in der wissenschaftlichen Literatur bereits stark thematisiert, die Identifikation von Determinanten der Attraktivität eines Arbeitgebers aus Sicht der aktuellen Mitarbeitenden im Unternehmen wurde bisher aber weitgehend vernachlässigt. Mit der Entwicklung eines Untersuchungsmodells zum Aufbau von Arbeitgeberattraktivität und dessen empirischer Überprüfung leistet der vorliegende Artikel einen ersten Beitrag dazu, diese Lücke zu schliessen.

Basierend auf den Ergebnissen der Untersuchung können folgende zentrale Erkenntnisse festgehalten werden: Mitarbeitende finden ihren Arbeitgeber insbesondere dann attraktiv, wenn „weiche“ Faktoren sowie Imagefaktoren als positiv wahrgenommen werden. Dies ist z.B. der Fall, wenn sich Mitarbeitende am Arbeitsplatz bzw. mit der Führung im Unternehmen wohlfühlen, der Arbeitgeber ihnen ein gewisses Prestige verschafft sowie ein attraktives Stellenprofil bietet. „Harte“ Faktoren, z.B. das Vorhandensein von Möglichkeiten der Weiterentwicklung oder die Entlohnung, tragen, der Studie zufolge, nicht zum Aufbau einer hohen Arbeitgeberattraktivität bei. Hier müsste sicherlich eine weitere Differenzierung erfolgen, z.B. hinsichtlich des Alters des Mitarbeitenden oder danach, ob sich der Mitarbeitende am Beginn oder am Ende der Berufslaufbahn befindet. Die Ergebnisse sind daher nicht als allgemeingültig anzusehen, vielmehr sind Replikationsstudien mit differenzierten Analysen notwendig.

Für die Unternehmen, die als attraktiver Arbeitgeber wahrgenommen werden möchten, ergeben sich folgende Implikationen: Die Personalführung ist so auszurichten, dass sie von den Mitarbeitenden als „arbeitnehmerfreundlich“ angesehen wird. Hier kommt den Füh- 
rungskräften eine besondere Bedeutung zu. Für Führungskräfte gilt es, einen Führungsstil zu praktizieren, der als positiv perzipiert wird. Weltweit durchgeführte Befragungen in Unternehmen führten zur Erkenntnis, dass über alle Kulturen hinweg folgende Führungsideale bestehen: Integrität, Inspiration, Leistungsorientierung, Vision, Teamintegration, Entschlossenheit, administrative Kompetenz und Diplomatie der Führungskraft (Dorfman et al. 2004). Die vorliegende Studie wurde im deutschsprachigen Kulturraum durchgeführt. Neben den weltweit geltenden Führungsidealen sind Führungskräfte zusätzlich dahingehend zu sensibilisieren, ihren Mitarbeitenden die Möglichkeit zur Partizipation (z.B. bei der Entscheidungsfindung) einzuräumen (Brodbeck et al. 2000). Die Einstellung der Führungskräfte gegenüber dem Arbeitgeber ist ebenfalls von Bedeutung. So nehmen Führungskräfte als „Role models“ Einfluss auf die Attraktivitätswahrnehmung der Mitarbeitenden (zu Role Models im organisationalen Kontext vgl. Gibson 2003). Führungskräfte sind zudem dahingehend zu schulen, dass sie ihren Mitarbeitenden gegenüber eine offene, transparente Informations- und Kommunikationspolitik betreiben, so dass der Entstehung von Informationsdefiziten auf Seiten der Mitarbeitenden begegnet werden kann. Im Hinblick auf die Bedeutung des Unternehmensimages ist ein „Impression Management“ von Seiten des Unternehmens dahingehend zu betreiben, dass die Mitarbeitenden ein positives Bild vom Unternehmen aufweisen und Stolz sind, für dieses zu arbeiten. Hierfür bietet sich die Durchführung interner Kommunikationskampagnen an, die den Mitarbeitenden das Arbeitgeberimage und die Employee Value Proposition klar kommunizieren und so eine interne Verankerung des Arbeitgeberimages garantieren. Insbesondere eignen sich hier Massnahmen, die über eine starke Emotionalisierungskraft verfügen, wie z.B. Social Events, Spiele, Geschichten über das Unternehmen als Arbeitgeber usw. Es ist darüber hinaus ein Management zur Sicherung bzw. Steigerung der Arbeitsplatzqualität zu betreiben, das z.B. eine angenehme Arbeitsatmosphäre, flexible Arbeitszeitmodelle und die Berücksichtigung der Work-Life-Balance der Mitarbeitenden sicherstellt (z.B. die Förderung von Sportaktivitäten). Die Schaffung von attraktiven Stellenprofilen, die den Mitarbeitenden, z.B. mittels Empowerment, Entscheidungsbefugnisse einräumt, wirkt sich zusätzlich fördernd auf die Attraktivitätswahrnehmung aus. Auch wenn in der vorliegenden Studie den Entwicklungsmöglichkeiten empirisch keine Bedeutung zukommt, ist dennoch auf das kontinuierliche Angebot von Schulungen, Job Rotations oder Weiterbildungen zu achten, da frühere Studien diesem Aspekt eine hohe Bedeutung zukommen lassen und der nicht erbrachte Beleg auf z.B. mitarbeiterspezifische Faktoren zurückgeführt wird. Gleiches gilt für die Entlohnung der Mitarbeitenden. Trotz des nicht nachgewiesenen Einflusses auf die Arbeitgeberattraktivität ist auf attraktive Entlohnungsmöglichkeiten zu achten.

Der vorliegende Artikel liefert einen Beitrag zur Identifikation der Determinanten der Arbeitgeberattraktivität aus Sicht von aktuellen Mitarbeitenden. Er unterliegt jedoch auch einigen Restriktionen, die der Generalisierbarkeit der gewonnenen Erkenntnisse entgegenstehen. Weiterer Forschungsbedarf eröffnet sich zum einen in methodischer Hinsicht. Die Stichprobengenerierung erfolgte durch das „Schneeball-Prinzip“. Dadurch besteht jedoch die Gefahr, dass sich die Merkmale des ersten (zufälligen) Schneeballs weiter verbreiten. Damit einher geht eine höhere Wahrscheinlichkeit einer homogenen Stichprobe im Vergleich zu auf andere Art gewonnene Samples und der damit verbundene Ausschluss weiterer Probanden (Browne 2005). Um eine repräsentative Stichprobe zu erhalten, ist daher im Rahmen künftiger Studien über alternative Verfahren zur Stichprobengenerierung nachzudenken. Zum anderen ist in inhaltlicher Hinsicht zu überprüfen, inwieweit die Kausalzu- 
sammenhänge von bestimmten Kriterien, wie z.B. Alter, Bildung, Stellung im Unternehmen, Arbeitsinhalt oder Branche, abhängig sind (vgl. Abschnitt 3.4). Die vorliegende Studie wurde unternehmensübergreifend durchgeführt. Forschungsbedarf besteht dadurch hinsichtlich der Berücksichtigung einzelner Unternehmen und deren Vergleich untereinander. Diese könnten z.B. nach kleinen, mittelständischen und Grossunternehmen klassifiziert und auf ihre Unterschiede hin untersucht werden. Aufgrund der internationalen Ausrichtung vieler Unternehmen ist zudem ein internationaler Vergleich hinsichtlich der Bedeutung der Einflussfaktoren der Arbeitgeberattraktivität in den einzelnen Ländern von Interesse. Schliesslich ist anzumerken, dass die Studie im Jahr 2009 durchgeführt wurde. $\mathrm{Zu}$ diesem Zeitpunkt befanden sich nahezu sämtliche Branchen in einer Krisensituation. Es besteht daher die Notwendigkeit weiterer Modellprüfungen, um zu untersuchen, inwieweit die Ergebnisse in Abhängigkeit des damaligen ökonomischen Umfelds zu betrachten sind.

\section{Literaturhinweise}

Arachchige, B.J.H./Robertson, A. (2011): Business Student Perceptions of a Preferred Employer: A Study Identifying Determinants of Employer Branding, in: IUP Journal of Brand Management, Jg. 8, Nr. 3, S. 25-46.

Axelrod, E.L./Handfield-Jones, H./Welsh, T.A. (2001): The war for talent, part two, in: McKinsey Quarterly, o. Jg., Nr. 2, S. 9-12.

Backhaus, K./Tikoo, S. (2004): Conceptualizing and researching employer branding, in: Career Development International, Jg. 9, Nr. 5, S. 501-517.

Bagozzi, R.P./Yi, Y. (1988): On the Evaluation of Structural Equation Models, in: Journal of the Academy of Marketing Science, Jg. 16, Nr. 1, S. 74-94.

Barrow, S./Mosley, R. (2006): Internes Brand Management, Weinheim.

Berthon, P./Ewing, M./Hah, L.L. (2005): Captivating company: Dimensions of attractiveness in employer branding, in: International Journal of Advertising, Jg. 24, Nr. 2, S. 151-172.

Böttger, E. (2012): Employer Branding. Verhaltenstheoretische Analysen als Grundlage für die identitätsorientierte Führung von Arbeitgebermarken, Wiesbaden.

Brinkmann, R. (2007): Ältere Beschäftigte. Einsatzfreude keine Frage des Alters, in: Böcklerimpuls, o. Jg., Nr. 17, S. 7, unter http://www.boeckler.de/pdf/impuls_2007_17_7.pdf, Zugriff: 2. November 2012.

Brodbeck, F.C., et al. (2000): Cultural variation of leadership prototypes across 22 European countries, in: Journal of Occupational \& Organizational Psychology, Jg. 73, Nr. 1, S. 1-29.

Browne, K. (2005): Snowball Sampling: Using Social Networks to Research Non-heterosexual Women, in: International Journal of Social Research Methodology, Jg. 8, Nr. 1, S. 47-60.

Burmann, C./Schäfer, K. (2007): Das Branchenimage als Determinante des Unternehmensimages, in: Die Betriebswirtschaft, Jg. 67, Nr. 1, S. 135-152.

Cable, D.M./Graham, M.E. (2000): The determinants of job seekers' reputation perceptions, in: Journal of Organizational Behavior, Jg. 21, Nr. 8, S. 929-947.

Chambers, E.G., et al. (1998): The war for talent, in: McKinsey Quarterly, o. Jg., Nr. 3, S. 44-57.

Chin, W.W. (1998a): Issues and opinion on structural equation modeling, in: MIS Quarterly, Jg. 22, Nr. 1, S. 7-16.

Chin, W.W. (1998b): The Partial Least Approach to Structural Equation Modeling, in: Marcoulides, G.A. (Hrsg.): Modern Methods for Business Research, Mahwah, S. 295-336. 
Chin, W.W./Newsted, P.R. (1999): Structural Equation Modeling Analysis with Small Samples Using Partial Least Squares, in: Hoyle, R.H. (Hrsg.): Strategies for Small Sample Research, Thousand Oaks, S. 307-347.

Chin, W.W./Peterson, R.A./Brown, S.P. (2008): Structural equation modeling in marketing - Some practical reminders, in: Journal of Marketing Theory and Practice, Jg. 16, Nr. 4, S. 278-298.

Churchill, G.A. (1979): A Paradigm for Developing Better Measures of Marketing Constructs, in: Journal of Marketing Research, Jg. 16, Nr. 1, S. 64-73.

Davies, G. (2008): Employer branding and its influence on managers, in: European Journal of Marketing, Jg. 42, Nr. 5/6, S. 667-681.

Diamantopoulos, A. (2005): The C-OAR-SE procedure for scale development in marketing: A comment, in: International Journal of Research in Marketing, Jg. 22, Nr. 1, S. 1-9.

Diamantopoulos, A./Winklhofer, H.M. (2001): Index construction with formative indicators: An alternative to scale development, in: Journal of Marketing Research, Jg. 38, Nr. 2, S. 269-277.

Dorfman, P./Hanges, P.J./Brodbeck, F.C. (2004): Leadership and cultural variation: The identification of culturally endorsed leadership profiles, in: House, R.J., et al. (Hrsg.): Culture leadership, and organizations: The GLOBE study of 62 societies, Thousand Oaks, S. 669-719.

Ewing, M.T., et al. (2002): Employment branding in the knowledge economy, in: International Journal of Advertising, Jg. 21, Nr. 1, S. 3-22.

Finn, A./Kayande, U. (2005): How fine is C-OAR-SE? A generalizability theory perspective on Rossiter's procedure, in: International Journal of Research in Marketing, Jg. 22, Nr. 1, S. 11-21.

Fornell, C./Larcker, D.A. (1981): Evaluating Structural Equation Models with Unobservable Variables and Measurement Error, in: Journal of Marketing Research, Jg. 18, Nr. 1, S. 39-50.

Forster, A./Erz, A./Jenewein, W. (2012): Employer Branding - Ein konzeptioneller Ansatz zur markenorientierten Mitarbeiterführung, in: Tomczak, T., et al. (Hrsg.): Behavioral Branding - Wie Mitarbeiterverhalten die Marke stärkt, 3. Aufl., Wiesbaden, S. 277-294.

Gaddam, S. (2008): Modeling Employer Branding Communication: The Softer Aspect of HR Marketing Management, in: ICFAI Journal of Soft Skills, Jg. 2, Nr. 1, S. 45-55.

Gibson, D.E. (2003): Developing the professional self-concept: role model construals in early, middle, and latent career stages, in: Organisation Science, Jg. 14, Nr. 5, S. 591-610.

Gillies, J.-M./Jung, A. (1999): Die 100 Besten Unternehmen, in: BIZZ Capital, o. Jg., Nr. 6, S. 21-32.

Great place to work® Institute (2012): Was ist eine ausgezeichnete Arbeitsplatzkultur?, unter http://www.greatplacetowork.de/unser-ansatz/was-bedeutet-ausgezeichnete-arbeitsplatz-kultur. Zugriff: 15. Oktober 2012.

Grobe, E. (2003): Corporate Attractiveness - eine Analyse der Wahrnehmung von Unternehmensmarken aus der Sicht von High Potentials, Arbeitspapier Nr. 50 der Handelshochschule Leipzig, Leipzig.

Grosse Halbuer, A. (2003): Erfolg macht sexy, in: Wirtschaftswoche, Jg. 33, S. 68-73.

Harman, H.H. (1967): Modern Factor Analysis, Chicago.

Heilmann, P.A.K. (2010): Employer brand image in a health care organization, in: Management Research Review, Jg. 33, Nr. 2, S. 134-144.

Herrmann, A./Huber, F./Kressmann, F. (2006): Varianz- und kovarianzbasierte Strukturgleichungsmodelle - Ein Leitfaden zu deren Spezifikation, Schätzung und Beurteilung, in: Zeitschrift für betriebswirtschaftliche Forschung, Jg. 58, Nr. 2, S. 34-66. 
Highbouse, S., et al. (1999): Assessing company employment image: An example in the fast food industry, in: Personnel Psychology, Jg. 52, Nr. 1, S. 151-172.

Hinzdorf, T./Priemuth, K./Erlenkämper, S. (2003): Präferenzmatching zur Steuerung des Employer Branding, in: Personal, Jg. 55, Nr. 8, S. 18-20.

Holtbrügge, D./Rygl, D. (2002): Arbeitgeberimage deutscher Grossunternehmungen, in: Personal, Jg. 54, Nr. 10, S. 18-21.

Homburg, Ch./Giering, A. (1996): Konzeptualisierung und Operationalisierung komplexer Konstrukte - Ein Leitfaden für die Marketingforschung, in: Marketing ZFP, Jg. 18, Nr. 1, S. 5-24.

Jarvis, C.B./Mackenzie, S.B./Podsakoff, Ph.M./Mick, D.G./Bearden, W.O. (2003): A critical review of construct indicators and measurement model misspecifications in marketing and consumer research, in: Journal of Consumer Research, Jg. 30, Nr. 2, S. 199-218.

Keller, K.L. (1993): Conceptualizing, Measuring, and Managing Customer-Based Brand Equity, in: Journal of Marketing, Jg. 57, Nr.1, S. 1-22.

Kirchgeorg, M./Günther, E. (2006): Employer Brands zur Unternehmensprofilierung im Personalmarkt, Arbeitspapier Nr. 74 der Handelshochschule Leipzig, Leipzig.

Knox, S./Freeman, C. (2006): Measuring and managing employer brand image in the service industry, in: Journal of Marketing Management, Jg. 22, Nr. 7/8, S. 695-716.

Krüger, D. (2012): Lufthansa: Mit Employer Branding die Richtigen finden, in: Tomczak, T., et al. (Hrsg.): Behavioral Branding - Wie Mitarbeiterverhalten die Marke stärkt, 3. Aufl., Wiesbaden, S. 317-334.

Liang, H., et al. (2007): Assimilation of Enterprise Systems: The Effect of Institutional Pressures and the Mediating Role of Top Management, in: MIS Quarterly, Jg. 31, Nr. 1, S. 59-87.

Lievens, F. (2007): Employer Branding in the Belgian Army: the Importance of Instrumental and Symbolic Beliefs for Potential Applicants, Actual Applicants, and Military Employees, in: $\mathrm{Hu}-$ man Resource Management, Jg. 46, Nr. 1, S. 51-69.

Lievens, F., et al. (2001): Organizational attractiveness for prospective applicants: A person-organisation fit perspective, in: Applied Psychology: An International Review, Jg. 50, Nr. 1, S. 30-51.

Lievens, F./Highbouse, S. (2003): The relation of instrumental and symbolic attributes to a company's attractiveness as an employer, in: Personnel Psychology, Jg. 56, Nr. 1, S. 75-102.

Lievens, F./Van Hoye, G./Anseel, F. (2007): Organizational Identity and Employer Image: Towards a Unifying Framework, in: British Journal of Management, Jg. 18, Nr. 1, S. 45-59.

Lievens, F./Van Hoye, G./Schreurs, B. (2005): Examining the relationship between employer knowledge dimensions and organizational attractiveness: An application in a military context, in: Journal of Occupational and Organizational Psychology, Jg. 78, Nr. 4, S. 553-572.

Maxwell, R./Knox, S. (2009): Motivating employees to "live the brand": a comparative case study of employer brand attractiveness within the firm, in: Journal of Marketing Management, Jg. 25, No. 9, S. 893-907.

Moroko, L./Uncles, M.D. (2008): Characteristics of successful employer brands, in: Journal of Brand Management, Jg. 16, Nr. 3, S. 160-175.

Moroko, L./Uncles, M.D. (2009): Employer branding, in: Wall Street Journal, Jg. 67, Nr. 253, Sp. R7.

Nunnally, J.C. (1978): Psychometric Theory, 2. Aufl., New York.

Petkovic, M. (2008): Employer Branding - Ein markenpolitischer Ansatz zur Schaffung von Präferenzen bei der Arbeitgeberwahl, 2. Aufl., Mering. 
Podsakoff, P.M., et al. (2003), Common Method Biases in Behavioral Research: A Critical Review of the Literature and Recommended Remedies, in: Journal of Applied Psychology, Jg. 88, Nr. 5, S. 879-903.

Rossiter, J.R. (2002): The C-OAR-SE procedure for scale development in marketing, in: International Journal of Research in Marketing, Jg. 19, Nr. 4, S. 305-335.

Roy, S.K. (2008): Identifying the Dimensions of Attractiveness of an Employer Brand in the Indian Context, in: South Asian Journal of Management, Jg. 15, Nr. 4, S. 110-130.

Schulze, R./Gürntke, K./Inglsperger, A. (2005): Employer Branding - Talente gewinnen, motivieren, binden, in: Absatzwirtschaft, Jg. 48, Nr. 1, S. 92-94.

Schwaab, M.-O. (2008): Die Bedeutung der sozialen Verantwortung für die Arbeitgeberattraktivität, in: Umwelt-Wirtschafts-Forum, Jg. 16, Nr. 4, S. 199-204.

Shah, M. (2011): Talent Retention through Employer Branding, in: Journal of Marketing \& Communication, Jg. 6, Nr. 3, S. 30-33.

Simon, H., et al. (1995): Effektives Personalmarketing - Strategien, Instrumente, Fallstudien, Wiesbaden.

Slaughter, J.E., et al. (2004): Personality trait inferences about organizations: Development of a measure and assessment of construct validity, in: Journal of Applied Psychology, Jg. 89, Nr. 1, S. 85-103.

Soutar, G.N./Clarke, A. (1983): Examining business students' career preferences: a perceptual space approach, in: Journal of Vocational Behavior, Jg. 23, Nr. 1, S. 11-21.

Stotz, W./Wedel, A. (2009): Employer Branding - Mit Strategie zum bevorzugten Arbeitgeber, München.

Sutherland, M.M./Torricelli, D.G./Karg, R.F. (2002): Employer-of-choice branding for knowledge workers, in: South African Journal of Business Management, Jg. 33, Nr. 4, S. 13-20.

Teufer, S. (1999): Die Bedeutung des Arbeitgeberimage bei der Arbeitgeberwahl: Theoretische Analyse und empirische Untersuchung bei High Potentials, Wiesbaden.

Tochtermann, T./Abend, J. (2003): War for Talents - Bedeutung und Ausrichtung des Talentmanagement, in: Hungenberg, H./Meffert, J. (Hrsg.): Handbuch Strategisches Management, Wiesbaden, S. 887-897.

Trost, A. (2008): Authentische Arbeitgebermarken, in: HR Today, o. Jg., Nr. 1, S. 6-7.

Turban, D.B./Keon, T.L. (1993): Organizational attractiveness: An interactionist perspective, in: Journal of Applied Psychology, Jg. 78, Nr. 2, S. 184-193.

Universum Communications AB (2012): Young Professionals wählen BMW, Audi und Porsche zu ihren beliebtesten Arbeitgebern, unter http://www.universumglobal.com/stored-images/8e/ 8e3951e1-e909-44b5-97f7-d383e3d789a5.pdf. Zugriff: 15. Oktober 2012.

Van Hoye, G./Saks, A.M. (2011): The Instrumental-Symbolic Framework: Organisational Image and Attractiveness of Potential Applicants and their Companions at a Job Fair, in: Applied Psychology: An International Review, Jg. 60, Nr. 2, S. 311-335.

Wiese, D. (2005): Employer Branding - Arbeitgebermarken erfolgreich aufbauen, Saarbrücken.

Wilden, R./Gudergan, S./Lings, I. (2010): Employer branding: strategic implications for staff recruitment, in: Journal of Marketing Management, Jg. 26, Nr. 1/2, S. 56-73.

Zinkhan, G.M./Burton, S./Wallendorf, M. (1983): Marketing Applications for Snowball Sampling: Potential Benefits and Problems, in: Darden, W.R./Monroe, K.B./Dillon, W.R. (Hrsg.): Research Models and Causal Modeling in Marketing, Chicago, S. 5-8.

Zirnsack, E. (2008): Employer Branding als Ausprägung des Personalmarketings, Saarbrücken. 


\section{Anhang}

\begin{tabular}{|c|c|c|c|c|}
\hline \multicolumn{5}{|l|}{ Determinanten der Arbeitgeberattraktivität } \\
\hline Arbeitsplatzqualität & $M W$ & $S D$ & $\begin{array}{l}\text { Faktor- } \\
\text { ladung }\end{array}$ & $\begin{array}{l}\text { T-Wert } \\
(>1,64)\end{array}$ \\
\hline $\begin{array}{l}\text { Ich finde, dass mein Arbeitgeber eine gu- } \\
\text { te Work-Life-Balance (Balance zwischen } \\
\text { Arbeit und Freizeit) ermöglicht. }\end{array}$ & 4,86 & 1,54 & 0,713 & 9,019 \\
\hline $\begin{array}{l}\text { Ich finde, dass bei meinem Arbeitgeber } \\
\text { ein gutes Arbeitszeitmodell (z.B. flexible } \\
\text { Arbeitszeit oder Blockzeiten) angewendet } \\
\text { wird. }\end{array}$ & 5,26 & 1,57 & 0,851 & 23,149 \\
\hline $\begin{array}{l}\text { Ich finde, dass mein Arbeitgeber einen } \\
\text { grossen Spassfaktor bietet. }\end{array}$ & 4,47 & 1,53 & 0,717 & 13,181 \\
\hline $\begin{array}{l}\text { Ich finde, dass mein Arbeitgeber eine ho- } \\
\text { he Arbeitsplatzsicherheit bietet. }\end{array}$ & 5,20 & 1,39 & 0,530 & 4,637 \\
\hline Konstruktreliabilität & \multicolumn{4}{|c|}{0,800} \\
\hline Durchschnittlich Erfasste Varianz & \multicolumn{4}{|c|}{0,507} \\
\hline Alpha & \multicolumn{4}{|c|}{0,669} \\
\hline Führungsstil & $M W$ & $S D$ & $\begin{array}{l}\text { Faktor- } \\
\text { ladung }\end{array}$ & $\begin{array}{l}\text { T-Wert } \\
(>1,64)\end{array}$ \\
\hline $\begin{array}{l}\text { Ich finde, dass die Vorgesetzten bei mei- } \\
\text { nem Arbeitgeber einen guten Führungs- } \\
\text { stil haben. }\end{array}$ & 4,68 & 1,53 & 0,907 & 45,005 \\
\hline $\begin{array}{l}\text { Ich finde, dass bei meinem Arbeitgeber } \\
\text { die Leistungen der Mitarbeitenden wert- } \\
\text { geschätzt werden. }\end{array}$ & 4,86 & 1,35 & 0,880 & 36,895 \\
\hline $\begin{array}{l}\text { Ich finde, dass die Vorgesetzten bei mei- } \\
\text { nem Arbeitgeber ein positives Vorbild } \\
\text { abgeben. }\end{array}$ & 4,86 & 1,34 & 0,883 & 34,540 \\
\hline $\begin{array}{l}\text { Ich finde, dass mein Arbeitgeber eine po- } \\
\text { sitive interne Informations- und Kommu- } \\
\text { nikationspolitik betreibt. }\end{array}$ & 4,69 & 1,48 & 0,808 & 25,934 \\
\hline Konstruktreliabilität & \multicolumn{4}{|c|}{0,926} \\
\hline Durchschnittlich Erfasste Varianz & \multicolumn{4}{|c|}{0,758} \\
\hline Alpha & \multicolumn{4}{|c|}{0,891} \\
\hline Entlohnung & $M W$ & $S D$ & $\begin{array}{l}\text { Faktor- } \\
\text { ladung }\end{array}$ & $\begin{array}{l}\text { T-Wert } \\
(>1,64)\end{array}$ \\
\hline $\begin{array}{l}\text { Ich finde, dass mein Arbeitgeber einen } \\
\text { guten (Einstiegs-)Lohn zahlt. }\end{array}$ & 4,48 & 1,63 & 0,823 & 23,599 \\
\hline $\begin{array}{l}\text { Ich finde, dass mein Arbeitgeber ein gu- } \\
\text { tes Lohnerhöhungspotenzial bietet. }\end{array}$ & 4,20 & 1,67 & 0,895 & 44,680 \\
\hline
\end{tabular}




\begin{tabular}{|c|c|c|c|c|}
\hline $\begin{array}{l}\text { Ich finde, dass mein Arbeitgeber eine gu- } \\
\text { te Erfolgsbeteiligung (z.B. in Form eines } \\
\text { Lohnbonussystems) bietet. }\end{array}$ & 3,38 & 1,81 & 0,764 & 14,542 \\
\hline $\begin{array}{l}\text { Ich finde, dass mein Arbeitgeber gute } \\
\text { Fringe Benefits (Lohnnebenleistungen } \\
\text { und überobligatorische Sozialleistungen) } \\
\text { bietet. }\end{array}$ & 4,25 & 1,85 & 0,848 & 31,504 \\
\hline Konstruktreliabilität & \multicolumn{4}{|c|}{0,901} \\
\hline Durchschnittlich Erfasste Varianz & \multicolumn{4}{|c|}{0,696} \\
\hline Alpha & \multicolumn{4}{|c|}{0,852} \\
\hline Stellenprofil & $M W$ & $S D$ & $\begin{array}{l}\text { Faktor- } \\
\text { ladung }\end{array}$ & $\begin{array}{l}\text { T-Wert } \\
(>1,64)\end{array}$ \\
\hline $\begin{array}{l}\text { Ich finde, dass meine Aufgabe bei mei- } \\
\text { nem Arbeitgeber interessant ist. }\end{array}$ & 5,26 & 1,47 & 0,915 & 59,644 \\
\hline $\begin{array}{l}\text { Ich finde, dass meine Aufgabe bei mei- } \\
\text { nem Arbeitgeber vielfältig ist. }\end{array}$ & 5,18 & 1,49 & 0,929 & 54,196 \\
\hline $\begin{array}{l}\text { Ich finde, dass meine Aufgabe bei mei- } \\
\text { nem Arbeitgeber herausfordernd ist. }\end{array}$ & 5,03 & 1,57 & 0,911 & 35,807 \\
\hline $\begin{array}{l}\text { Ich finde, dass ich bei meinem Arbeitge- } \\
\text { ber ein gutes Mass an Entscheidungsbe- } \\
\text { fugnis habe. }\end{array}$ & 4,84 & 1,55 & 0,797 & 17,661 \\
\hline Konstruktreliabilität & \multicolumn{4}{|c|}{0,938} \\
\hline Durchschnittlich Erfasste Varianz & \multicolumn{4}{|c|}{0,791} \\
\hline Alpha & \multicolumn{4}{|c|}{0,910} \\
\hline Entwicklungsmöglichkeiten & $M W$ & $S D$ & $\begin{array}{l}\text { Faktor- } \\
\text { ladung }\end{array}$ & $\begin{array}{l}\text { T-Wert } \\
(>1,64)\end{array}$ \\
\hline $\begin{array}{l}\text { Ich finde, dass mein Arbeitgeber gute } \\
\text { Aus- und Weiterbildungsmöglichkeiten } \\
\text { bietet. }\end{array}$ & 4,78 & 1,74 & 0,858 & 26,865 \\
\hline $\begin{array}{l}\text { Ich finde, dass mein Arbeitgeber gute } \\
\text { Karrieremöglichkeiten bietet. }\end{array}$ & 4,50 & 1,59 & 0,859 & 32,899 \\
\hline $\begin{array}{l}\text { Ich finde, dass mein Arbeitgeber gute } \\
\text { Möglichkeiten bietet, innerhalb des Un- } \\
\text { ternehmens den Job zu wechseln und } \\
\text { verschiedene Bereiche des Unternehmens } \\
\text { kennenzulernen. }\end{array}$ & 4,23 & 1,76 & 0,616 & 8,321 \\
\hline $\begin{array}{l}\text { Ich finde, dass mein Arbeitgeber gute } \\
\text { Lernchancen bietet. }\end{array}$ & 4,97 & 1,47 & 0,839 & 19,731 \\
\hline Konstruktreliabilität & \multicolumn{4}{|c|}{0,875} \\
\hline Durchschnittlich Erfasste Varianz & \multicolumn{4}{|c|}{0,639} \\
\hline Alpha & \multicolumn{4}{|c|}{0,802} \\
\hline
\end{tabular}




\begin{tabular}{|c|c|c|c|c|}
\hline Unternehmensimage & $M W$ & $S D$ & $\begin{array}{l}\text { Faktor- } \\
\text { ladung }\end{array}$ & $\begin{array}{l}\text { T-Wert } \\
(>1,64)\end{array}$ \\
\hline $\begin{array}{l}\text { Ich finde, dass mein Arbeitgeber ein er- } \\
\text { folgreiches Unternehmen ist. }\end{array}$ & 5,48 & 1,22 & 0,666 & 11,966 \\
\hline $\begin{array}{l}\text { Ich finde, dass mein Arbeitgeber ein po- } \\
\text { sitives Unternehmensimage hat. }\end{array}$ & 5,37 & 1,27 & 0,791 & 16,216 \\
\hline $\begin{array}{l}\text { Ich finde, dass die Branche, in der mein } \\
\text { Arbeitgeber tätig ist, ein positives Image } \\
\text { hat. }\end{array}$ & 4,95 & 1,32 & 0,464 & 4,779 \\
\hline $\begin{array}{l}\text { Ich finde, dass die Produkte/Dienstleis- } \\
\text { tungen, die mein Arbeitgeber produziert, } \\
\text { ein positives Image haben. }\end{array}$ & 5,39 & 1,14 & 0,627 & 7,828 \\
\hline $\begin{array}{l}\text { Ich finde, dass mein Arbeitgeber einen } \\
\text { guten Standort hat. }\end{array}$ & 5,66 & 1,48 & 0,474 & 4,412 \\
\hline $\begin{array}{l}\text { Ich finde, dass mein Arbeitgeber einen } \\
\text { guten Grad an Internationalität hat. }\end{array}$ & \multicolumn{4}{|c|}{ Wurde aufgrund geringer Faktorladung eliminiert. } \\
\hline $\begin{array}{l}\text { Ich finde, dass mein Arbeitgeber eine po- } \\
\text { sitive Unternehmenskultur und positive } \\
\text { Unternehmenswerte vertritt. }\end{array}$ & 5,21 & 1,37 & 0,829 & 26,739 \\
\hline $\begin{array}{l}\text { Ich finde, dass mein Arbeitgeber seine } \\
\text { Corporate Social Responsibility (soziale } \\
\text { und umweltpolitische Verantwortung } \\
\text { des Unternehmens) gut wahrnimmt. }\end{array}$ & 5,01 & 1,56 & 0,656 & 9,348 \\
\hline $\begin{array}{l}\text { Ich finde, dass mein Arbeitgeber einen } \\
\text { guten Bekanntheitsgrad hat. }\end{array}$ & \multicolumn{4}{|c|}{ Wurde aufgrund geringer Faktorladung eliminiert. } \\
\hline $\begin{array}{l}\text { Ich finde, dass mein Arbeitgeber gegen- } \\
\text { über seinen Mitarbeitenden ein glaub- } \\
\text { würdiges Unternehmen ist. }\end{array}$ & 5,28 & 1,31 & 0,836 & 26,459 \\
\hline Konstruktreliabilität & \multicolumn{4}{|c|}{0,869} \\
\hline Durchschnittlich Erfasste Varianz & \multicolumn{4}{|c|}{0,465} \\
\hline Alpha & \multicolumn{4}{|c|}{0,824} \\
\hline Arbeitgeberattraktivität & $M W$ & $S D$ & $\begin{array}{l}\text { Faktor- } \\
\text { ladung }\end{array}$ & $\begin{array}{l}\text { T-Wert } \\
(>1,64)\end{array}$ \\
\hline $\begin{array}{l}\text { Ich arbeite gerne bei meinem Arbeitge- } \\
\text { ber. }\end{array}$ & 5,41 & 1,30 & 0,861 & 32,359 \\
\hline $\begin{array}{l}\text { Ich habe gegenüber meinem Arbeitgeber } \\
\text { eine positive Einstellung. }\end{array}$ & 5,47 & 1,21 & 0,902 & 53,872 \\
\hline $\begin{array}{l}\text { Ich bevorzuge meinen Arbeitgeber gegen- } \\
\text { über anderen, vergleichbaren Arbeitge- } \\
\text { bern (Konkurrenten). }\end{array}$ & 5,14 & 1,36 & 0,787 & 13,575 \\
\hline
\end{tabular}




\begin{tabular}{|l|c|c|c|c|}
\hline $\begin{array}{l}\text { Ich fühle mich meinem Arbeitgeber emo- } \\
\text { tional verbunden. }\end{array}$ & 4,18 & 1,66 & 0,719 & 14,523 \\
\hline $\begin{array}{l}\text { Ich finde, dass mein Arbeitgeber attrak- } \\
\text { tiv ist. }\end{array}$ & 4,91 & 1,33 & 0,863 & 31,262 \\
\hline Konstruktreliabilität & \multicolumn{3}{|c|}{0,916} \\
\hline Durchschnittlich Erfasste Varianz & \multicolumn{3}{|c|}{0,687} \\
\hline Alpha & \multicolumn{3}{|c|}{0,877} \\
\hline Legende: MW = Mittelwert; SD = Standardabweichung
\end{tabular}

Tabelle 2: Ergebnisse der Messmodelle für die Modellkonstrukte

Manfred Bruhn, Prof. Dr., ist Inhaber des Lehrstuhls für Marketing und Unternehmensführung an der Wirtschaftswissenschaftlichen Fakultät der Universität Basel und Honorarprofessor an der Technischen Universität München.

Verena Batt, Dr. rer. pol., ist wissenschaftliche Mitarbeiterin am Lehrstuhl für Marketing und Unternehmensführung an der Wirtschaftswissenschaftlichen Fakultät der Universität Basel.

Benjamin Flückiger, Master of Science in Business and Economics, ist freier wissenschaftlicher Mitarbeiter am Lehrstuhl für Marketing und Unternehmensführung an der Wirtschaftswissenschaftlichen Fakultät der Universität Basel.

Anschrift: Universität Basel, Wirtschaftswissenschaftliche Fakultät, Lehrstuhl für Marketing und Unternehmensführung, Peter Merian-Weg 6, CH-4002 Basel, Tel.: +41 (0)61/267-32-22, Fax: +41 (0)61/267-28-38, E-Mails: manfred.bruhn@unibas.ch, verena.batt@unibas.ch 